\title{
Methodology Integration in Human Medical Study ${ }^{\dagger}$
}

\author{
Yixin Zhong \\ School of Computer, University of Posts and Telecommunications, Beijing 100876, China; zyx@bupt.edu.cn; \\ Tel.: +86-10-6228-1262 \\ † Presented at the IS4SI 2017 Summit DIGITALISATION FOR A SUSTAINABLE SOCIETY, Gothenburg, \\ Sweden, 12-16 June 2017.
}

Published: 9 June 2017

\begin{abstract}
Due to numerous factors two categories of methodology in scientific studies have been established till the present time, one for classical physics and the other for information science, each of which have been successful in its own domain. On the other hand, however, when dealing with the complex problems the cooperation and integration between the two categories of methodology will become an unavoidable necessary. Taking the human medical study as an example of complex problems, the necessity of methodological integration will be discussed in the paper.
\end{abstract}

Keywords: methodology; integration; reductionism; information ecology

\section{Introduction}

Methodology is commonly accepted as the general research strategy that outlines the way in which research is to be undertaken and identifies the methods to be used in it (although it does not define specific methods) [1].

More specifically, methodology is the embodiment of worldview and serves as the general guidelines for practical research. What worldview is possessed will determine what methodology being taken, so what scientific view is hold will determine what methodology of scientific study being employed. If the right methodology is taken to conduct research works, various challenges can better be resolved and remarkable progresses in research works can be produced; if the improper methodology is taken, the studies will be set back or failed.

Therefore, methodology to be employed is a very critical issue to whether a scientific study could be successful or not although one cannot clearly see, hear, or touch, it. This will be particularly true when complex sciences, like the biological systems study, the human medicine study, and the information and intelligence systems study, and so on, are under consideration.

\section{Two Scientific Views and Two Methodologies}

Information and matter are basic objects of scientific research. Looking back into the history of science development, it is recognized that the research object has been expanded from physical matter to information. With the expansion of research object, a new scientific view and scientific methodology have also grown up correspondingly. Thus two scientific views and two methodologies have coexisted for the time being. Of the two, one is the elder scientific view and methodology growing up from the study of matter and the systems of matter since the 17th century; and the other is the new scientific view and methodology growing up from the study of information and information systems roughly since the mid-20th century.

Due to the feature of matter as research objects and the human knowledge in modern time, the elder scientific view and methodology formed, more or less, a mechanically materialistic view of the world whereas due to the feature of information and the human knowledge in contemporary time, the new scientific view and methodology reflects, to a certain extent, the attitudes of dialectical 
materialism. It is really a great historical progress on scientific view and methodology from the elder to the new.

Roughly speaking, some of the major features for the two categories of scientific view and methodology are listed as that in Table 1 below.

Table 1. Brief Comparison between the Scientific Views and Methodologies.

\begin{tabular}{c|c|c|}
\hline Disciplines Compared & Classical Physical Science & Information Science \\
\hline Scientific View & Mechanical Materialism & Dialectic Materialism \\
\hline Feature 1 & Matter & Information \\
\hline Feature 2 & Static & Dynamic \\
\hline Feature 3 & Isolation & Interaction \\
\hline Feature 4 & Deterministic & Non-deterministic \\
\hline Methodology & Mechanical Reductionism & Information Ecology \\
\hline Feature 1 & Divide and Conquer & Entirety and Conversion \\
\hline
\end{tabular}

As can be seen from Table 1 that for understanding different discipline (classical physical science, information science) the different scientific view is needed and that different scientific view will in turn lead to different methodology. The two categories of scientific view and methodology for the two disciplines are quite different from each other and can hardly be exchanged, or replaced. More analyses on the features of the two categories of scientific view and methodology will respectively be given in next two sections

\section{The Scientific View and Methodology in the Study of Physical Matter System}

The primary scientific view in physical matter study is the physical matter view. According to this view, it holds that the objective world is composed of physical matter systems. The purpose of studying physical matter systems is to explicate physical matters' structures and, based on the understanding of them, to expound on various properties and functions of those systems.

Under the guidance of physical matter view, the research methods of physical matter system exploit various means to investigate the structure of physical matter system from the macro level to the micro levels. It is believed that only when the micro- structure of a physical matter system is clarified, can its properties and functions be explained and then the physical matter system could be understood. For a complicated physical matter system, it is requested being divided into a few simpler sub-systems and then, one by one, being observed and investigated with specific theoretical methods and experimental means to clarify their macro and micro-structures. In terms of scientific view and methodology, the structure, properties and functions of a complex system can be elucidated as long as the structures, properties and functions of all its sub-systems have been thoroughly comprehended. This methodology is thus named as "divide and conquer", or "reductionism" [2,3]. The basic feature of the methodology is dividing for analysis. Directed by the method of "divide and conquer", the study area of physical matter has constantly been divided into smaller and smaller parts. As results, more and more new research branches and disciplines in the study of physical matter were boosted, finer and deeper progresses in the study were produced, and more and more profound understandings on the physical world were achieved. All these contributions led the modern sciences and technology to the great prosperity never seen before.

Some of the major characteristics of "divide-and-conquer" methodology for the study of physical matter can be mentioned as follows.

Individuality: This scientific view of the methodology deems that the world is composed of various physical matter systems and the methods employed are to study them individually. Whenever a physical matter system is studied, the attention of study is concentrated on it while other physical matter systems are seldom considered. If a system is complicated enough, it will be divided into a number of sub-systems, and the attention of study will exclusively be given to each of the subsystem respectively with seldom consideration of the influence from other sub-systems. 
Isolation: When various physical matter systems (or the sub-systems) are investigated, more often than not, the exploration goes directly into the one under study paying no attention to any other systems (sub-systems) to get rid of the complicated interaction among the systems (sub-systems). Moreover, the human researcher's factors are also strictly excluded. Thus the research into the one concerned can be done profoundly.

Statics: In the scientific methodology for the study in physical matter, the structure, properties and functions of an object under investigation are treated as in static condition. At least during the time span when the study is conducted, they are considered static.

Determinism: according to scientific methodology for the study in physical matter, the structure, properties and functions of the object under investigation are of definite determinacy. Of them, Laplace Determinism is the representative.

It should be pointed out that for the physical matter systems study, the methodology of "divide and conquer" is effective. Benefiting from the methodology, humans' understanding of various physical matter systems actually keeps deepening.

\section{The Scientific View and Methodology for the Study in Information System}

Information system is dominantly featured by information and its dynamics. Although information system cannot be completely separated with physical matter and energy, but the major role that physical matter and energy play in an information system is to support to, and serve for, the information and its dynamics.

Information system is usually an open system and interacts with the outside world: it receives information from the outside world on one hand and on the other hand generates its intelligent action on the outside world [4]. For instance, human brain is an open complicated information system. It constantly receives relevant information from outside world on one hand and interacts with the outside world through its intelligent action on the other hand. In fact, the interactions between humans (the most typical information system) and the outside world are taking place, all the time and all the place, to implement human's purpose of continuously understanding the world and properly coping with it.

It is obvious that the objectives and methods for the study of information system are distinct from those used for the study of physical matter and its system. The purpose of research in information system is not to explain its matter structure and properties, but rather to study how it should generate proper strategy to interact with the outside world: when dealing with the information received from outside world, it should be able to produce the intelligent strategy so as to properly act on the outside world. That is to say that the radical purpose of research in information system is to accomplish the conversion of "information perceived from the outside world" into "the intelligent strategy generated by information system". This process has been generally termed as information conversion [5].

As for the medical study over human body is concerned, a practitioner's purpose is to give a proper medical strategy for treatment so as to make the patient recovered based on the patient's information obtained.

How is the information system able to produce proper action strategy after collecting the information from the object in outside world? It is well known that "information" can merely express the phenomena of object's dynamic motion while "knowledge" can actually represents the essence of object's dynamics. So, after receiving the information from the object in outside world, the system should firstly process it and convert it to knowledge for getting insights into the nature of the object in outside world. Only based on the knowledge, could the proper strategy (the main embodiment of intelligence) be deducted for dealing with the object in outside world. Thus, the steps stated above are in fact the "information-knowledge-intelligence" conversion.

All in all, the scientific view for the study of open and complex information system is information view rather than physical matter view. The view of information focuses on information instead of matter, on how information can be gradually grown up to the intelligent strategy within the framework of subject-object interaction (rather than the matter structure). 
Based upon the discussions above, the core of information science study is to implement the "information-knowledge-intelligence" conversion, or more specifically, to fulfill the conversion from object information to perceived information and then to knowledge and further to intelligent strategy and finally to intelligent action. This series of conversion does form a kind of ecological process as is seen in Figure 1 [6].

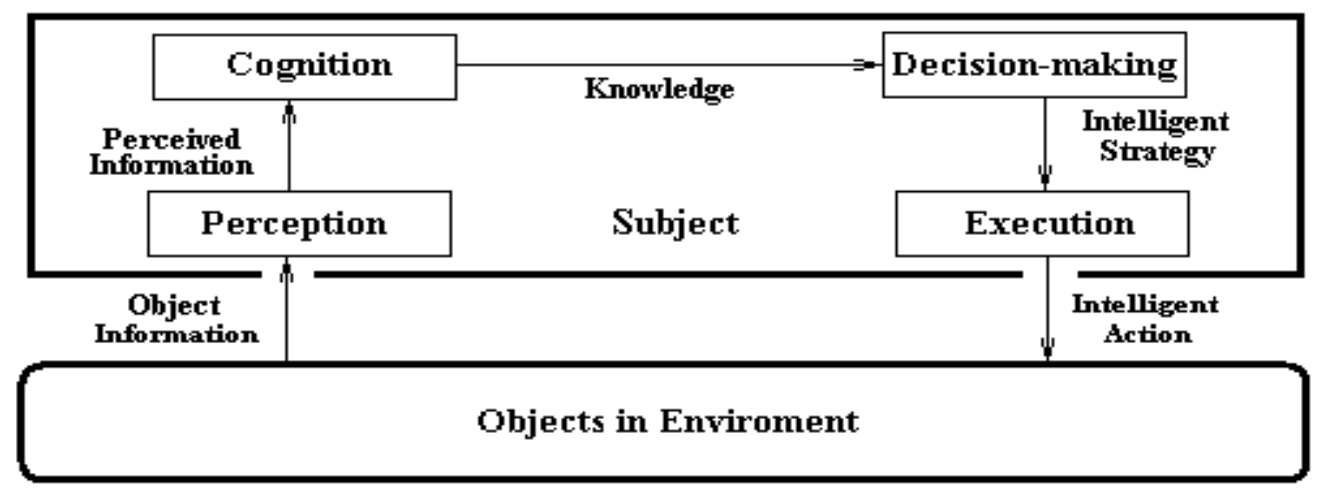

Figure 1. Model of Ecological Process in Information Study.

Note that the term "ecology" was coined by Ernst Haeckel ${ }^{[7]}$ and that refers to the scientific analysis and study of interactions among organisms and their environment for better living, This is an important, and fundamental, concept in scientific research, particularly in the fields of living systems and information systems.

In our case here, the methodology for information science research, the study of the interactions among the object information, the perceived information, the knowledge, the intelligence and their environment can then reasonably be defined as "Information Ecology" [8].

Note also that there have been some different explanations on the concept of information ecology. For example, Eryomin pointed out that information ecology is a science which studies the laws governing the influence of information summary on the formation and functioning of biosystems, including that of individuals, human communities, and humanity in general and on the health and psychological, physical and social well-being of the human being; and which undertakes to develop methodologies to improve the information environment [9]. Obviously, This explanation on the concept of information ecology is, more or less, right but it did not grasp the conversion of "information-knowledge-intelligence". It is this conversion that embodies the growth chain of information ecology.

Another explanation on information ecology is given by Por. He pointed out that information ecology makes a connection to the concept of collective intelligence and knowledge ecology [10]. This understanding is also right to a certain extent because it mentioned the components of "knowledge" and "intelligence". But it did not give the exact chain of information growing to knowledge and then to intelligence.

Therefore, comparing the definition and explanation on the concept of information ecology given in this paper with the references [9,10], our definition is not only more concise and more precise than the others but also more rational.

According to the analysis from reference [4], some of the primary features of the methodology of information Ecology are summarized as follows:

Integrity: From obtaining the information from the outside world to producing right action strategy, the information system implements a series of information conversion. All the processes form a systematic unity. Ultimately, whether an action strategy is appropriate is determined by the whole system rather than any one part or parts of it.

Interaction: From receiving the outside environment information to producing right action strategy, the series of information conversion implemented are mutually linked and interacted with 
one another, instead of isolated; the information system per se keeps linking to, and interacting with, the outside world as well.

Dynamics: The series of information conversion carried on in information system remains dynamic. Unavoidably, the state of the whole information system remains in a constantly changing rather than static one.

Complexity: When will an information system obtain outside-world information? What kind of information will it gain from the outside world? What would the interference with the information be received? The study in the open complicated information system is full of various uncertainties. How the outside information, associated with the relevant knowledge and the goal for the system, is processed to produce the right strategy? The research into all the issues is very tangled. Further, all the processes for the implementation of the information conversion are supported by physical systems and energy systems. So, there is the relationship among information, energy, and matter to be handled.

Abstraction: Compared with the physical matter, information is an entity that is more abstract to be studied. In view of this, the dynamics of information and its conversions are also relatively abstract. However, the ultimate effect from the abstract information and its conversion can be technically implemented and practically utilized. Hence, both primary scientific views and methodologies mentioned above are really precious treasure of human's society.

\section{The Methodology in Human Body Research}

Anyone will agree that, in the study of human body, the first issue to be clarified is what human body is. The paper holds that human body is not only a complicated physical matter system but also a complex information system as well. What is more, it is an open, sophisticated and dynamic system in which the physical matter and the information are mutually interacted to each other.

Western medicine treats human body mainly as a physical matter system, and thus it employs the methodology of "Divide and Conquer" or reductionism. It has developed and applied numerous of devices and instruments for its needs. From macro view to micro one, and from organs, to tissues, cells, molecules, and genes, it studies parts of human body including energy generation and transmission, targets, pathways, and detailed mechanism of protein functions, and manages to set up the criteria for physical matter structures of human body. It focuses on human's diseases and restores the abnormal physiological or pathological conditions by identifying them in patients' physical structures and energy processing.

TCM, on the other hand, sees human body as an open information system (though the term of information was not formally used before). By the use of dynamic view, holistic view, and systematic view, it grasps the states of physical dynamics presented in space, and the courses and patterns in time by observation, deduction, and by seeking the rationale behind them. TCM focuses on the patients themselves by treating each individual as a whole in terms of biology, psychology, and sociology. TCM employs the methodology of information conversion on the basis of human body system as a whole (although it may not be understood thoroughly). By inspecting, listening and smelling, questing, pulse taking and palpation, the information about the patient's body is collected and by pattern differentiation for treatment the intervention strategies are generated for the patient [11-14].

TCM's pattern differentiation for treatment is exactly a vivid paradigm of the information methodology. With patients centered, pattern differentiation for treatment is a holistic, dynamic, personalized, and complicated intervention process of focusing on regulating patient's conditions by closely combining patterns, interventions and effectiveness altogether. The effectiveness refers to the treatment effect evaluation covering the patient's both objective clinical symptoms and subjective feelings. Focusing on yin-yang balance, TCM sets up the direct and complicated correlation between intervention and its physician as the host, and the functional conditions of the patient as the guest. "Pattern" is used to describe and summarize the guest's dynamic state under the guidance of TCM theory. That means the state and trend of a guest's dynamics in space, namely, a guest's clinical manifestations - the "symptoms and signs" are described and summarized; the "symptoms and 
signs with coherence" in terms of TCM theory is the description and summarization of the guest's dynamics changes, that is, they highlight and depict the processes and patterns of the guest's dynamic states manifesting in time. The "pattern" and the "symptoms and signs with coherence" simply add up to "the pattern, and symptoms and signs with coherence". It highlights the guest's dynamic state in a specific time and space, which possesses the character of time-space dynamics.

The complicated process of intervention in TCM may be depicted with the model of cybernetics which says that, in order to control an entity (to improve the functions or development of an entity under study), information and knowledge must be obtained for generating actions on it. In view of this, the basis of controlling is information and knowledge. All information and knowledge transmitted and extracted are used for controlling, and any controlling processes to be realized depend on the information feed-forward or feedback. It refers to the information transmitted out and the outcome of an action fed back by controlling system, which affects the information that is retransmitted out to achieve constrain effect in order to achieve the preset goals. In the process of TCM's pattern differentiation for treatment, "pattern" is the entity under control, effectiveness is the key feedback information in the model of cybernetics; treatment is a mechanism that takes action on the entity under control. With patterns, interventions, and effectiveness closely combined, the medications are constantly administered so as to make human body's condition take a turn for the better. With the knowledge accumulated, TCM has established its own medical system.

It is clear from the descriptions above that the human body study is unable to be successful with either only the methodology of physical matter view-analyzing by dividing, or merely the methodology of information view and information conversion. The two methodologies should be effectively integrated to fulfill the goal of their tacit complement. The comprehensive understanding of human body should be supported and deepened by the body's micro and macro information; obtaining and processing the micro and macro information should be governed and guided by the comprehensive understanding of the body.

As once mentioned earlier in the paper, the methodology of information conversion is a systematic one. It aims to systematically and interactively study the issues in question; in contrast, the method of analyzing by dividing is the one that confines study to one part or parts of the whole issue under study. It intends to partially study the issue in statics (though the study may go deeper). Obviously, both methodologies have their strengths themselves and they should be efficiently integrated.

Then, is it possible to integrate the two methodologies? The answer is most likely yes. They are not incompatible like water and fire. In 1960s, the study in the integration of TCM with the Western medicine was proposed in China. Actually it was very reasonable and far-sighted. From methodological point of view, they can well be integrated for the two themselves are complementary.

The question is how to do it? In light of the basic purpose of human body study and the respective characters of the two methodologies, we deem that the methodology of information science for information conversion should play the role of framework while the methodology of physical matter science for analyzing by dividing should play the role of supplementation of the framework. This is because of the fact that the goal of human body study is to generate the strategy for the intervention of patients based on their information collected (If no any sick conditions are identified, the strategy should be treated as the suggestion for health promotion and disease prevention). This is what the methodology of information conversion does. Thus the methodology of information science should be treated as the framework. Nevertheless, the prerequisite for the methodology of information science performing is that the patients' information should be collected accurately and completely (including both the micro and the macro information). Furthermore, to extract the "knowledge" of patient's health conditions, the obtained "information" of the body should be processed and the relevant intervention strategy should be generated. And these are the important roles the methodology of analyzing by dividing can play.

Some may consider that the Western medicine should take a leading role while TCM be the follower in the practice of the integration of the two medicines. Their argument is that the technologies for diagnosis and treatment in the Western medicine are more advanced, which actually are underpinned by means of contemporary science and technology; on the contrary, those of TCM 
are more primitive and lack the supports from the modern science and technology. Its practice of diagnosis and treatment roughly rely merely on the ability of physicians themselves.

Admittedly these facts do exist, but from the perspectives of the purpose of human body study and the natures of the two methodologies, the conception should be well observed that the macro holistic view plays the leading role while the micro view does a supplementary one. This is the primary principle.

TCM still cannot sleep without any worries. In contrast, the integration of the methodology of information science and the methodology of physical matter science makes TCM face heavier tasks: TCM should take great efforts to keep up with modern science and technology (especially information science technology and intelligent technology) in order to precisely and fully capture human body information; it at the same time should also manage to theorize its practice experience accumulated over millennia; great endeavor should also be taken to explore and understand the mechanism of information-knowledge-intelligence conversion behind the human body: an open, dynamic, and complex system in which physical matters and information are interacted. The theory and knowledge obtained should be used in clinical practice to resolve problems and they should also be tested constantly and improved. It is a task of no easy. On the other hand, based on the advanced technologies and the advantages of its micro-view knowledge, the Western medicine should try hard to grasp the macro-view knowledge of open information system of human body.

It is certain that the integration of the two medicines should be more scientific, deeper, and more comprehensive and efficient than using any one of them alone. With the two methodologies efficiently integrated, doctors and physicians will not only have sufficient information for diagnosis, but also have deep understanding of the human body as an entire system from macro view and theoretic grounds for information conversion. These will enable human body research to make greater progresses that are the fruit TCM and the Western medicine produce together.

In fact, the study of human body has been advanced up to the present day. Both TCM and the Western medicine are faced with enormous challenges. It would be believed helpless if they develop independently. As the two methodologies have not been consolidated, amalgamated, integrated efficiently so far, TCM and its counterpart have undergone quite a few confusions. Nevertheless, if great effort is taken to efficiently integrate the two methodologies harmoniously, then either TCM or the Western medicine may proceed with great rapidity. In so doing, the unknown of human body can be expected to become less and less and the known more and more. Of course, it is absolutely impossible to exhaust all knowledge of human body within limited time for our environments are constantly changing and human body keeps changing as well. However, as long as the right scientific views and methodologies are established and employed, the available knowledge of human body can be constantly expanded, deepened, and better research results will be continuously achieved so as to better secure the health of human race.

\section{Conclusions}

We are now facing more or more complex problems in reality. Human medical study is one of the typical examples of this kind problem. The methodology of reductionism in classical physical science research and the one of information ecology in information science research should evidently be cooperatively applied.

Considering the fact that the methodology of information ecology is in agreement with the system thinking and that Eastern people are accustomed to it, the needs for cooperation between the methodology of reductionism and that of information ecology, might also reflect the needs of EastWest cooperation.

Conflicts of Interest: The authors declare no conflict of interest. 


\section{References}

1. Howell, K.E. Introduction to the Philosophy of Methodology; Sage Publication: New York, NY, USA, 2013.

2. Polkinghorne, J. "Reductionism". Interdisciplinary Encyclopedia of Religion and Science, Advanced School for Interdisciplinary Research; Pontifical University of The Holy Cross: Roma, Italy, 2002.

3. Jones, R.H. Reductionism: Analysis and the Fullness of Reality; Bucknell University Press: Lewisburg, PA, USA, 2000; pp. 24-26, 29-31.

4. Zhong, Y.X. Principles of Information Science; BUPT Press: Beijing, China, 1988.

5. Zhong, Y.X. Information Conversion: An Integrated Theory for Information, Knowledge, and Intelligence. Sci. Bull. 2013, 58, 1300-1306.

6. Zhong, Y.X. Information Conversion with Intelligence Creation: The Law that Governs the Information Discipline. Int. J. Cogn. Inf. Nat. Intell. 2015, 9, 25-41.

7. Stauffer, R.C. Haeckel, Darwin and Ecology. Q. Rev. Biol. 1957, 32, 138-144.

8. Zhong, Y.X. Principles of Advanced Artificial Intelligence; Science Press: Beijing, China, 2014.

9. Eryomin, A. Information Ecology-A viewpoint. Int. J. Environ. Stud. 1998, 54, 241-253.

10. Por, G. Nurturing Systemic Wisdom through Knowledge Ecology. Syst. Think. 2000, 11, 1-5.

11. Baoyan, L. The framework for real-world clinical study of Traditional Chinese Medicine. J. Tradit. Chin. Med. 2013, 6, 451-456.

12. Caozhong, Z. The issue of the reality of framework for Traditional Chinese Medicine: Commentary on the framework for real-world clinical study of Traditional Chinese Medicine. J. Tradit. Chin. Med. 2013, 14, 1171-1174.

13. Xinrong, H. Complicated science and Traditional Chinese Medicine. J. Tradit. Chin. Med. 2013, 19, $1621-1626$.

14. Tong, W. The body, space, and technology of Traditional Chinese Medicine and the Western medicine in medical practice: The difference of medical models between the two medicines in terms of human body. J. Tradit. Chin. Med. 2013, 22, 1891-1895. 